\title{
Quantifying the Ionising Flux escaping from the Ultra-luminous HII Regions in Four Spiral Galaxies
}

\author{
M. Rozas, A. Zurita and J. E. Beckman \\ Instituto de Astrofísica de Canarias, La Laguna, Spain 38200 \\ mrozas, azurita, jeb@ll.iac.es
}

\begin{abstract}
There are two principal problems in explaining, in terms of the emission from $\mathrm{OB}$ stars, the ionisation of gas which emits diffuse $\mathrm{H} \alpha$ in spiral galaxies. One is the long pathlength which the ionising photons need to traverse to reach their objectives, the other is whether sufficient photons can escape from within the H II regions which surround the hot stars. Here we treat the second point, assuming that the H II regions above a certain threshold luminosity are density bounded. We calculate the escaping Lyman continuum (Lyc) fluxes from the density bounded regions in four galaxies, and show that in each of them this is easily sufficient to produce the measured diffuse $\mathrm{H} \alpha$ emission.
\end{abstract}

Keywords: galaxies: ISM — galaxies: spiral — ISM: H II regions

\section{Observations}

All the galaxies were observed through the TAURUS camera on the $4.2 \mathrm{~m}$ William Herschel Telescope, La Palma. Calibrated photometric sub-arcsec images in appropriately red-shifted $\mathrm{H} \alpha$ were obtained by subtracting a nearby continuum image from that through the $\mathrm{H} \alpha$ filter. Absolute fluxes were determined via distances derived using $\mathrm{H}_{0}=75 \mathrm{~km}$ $\mathrm{s}^{-1} \mathrm{Mpc}^{-1}$.

\section{Data Analysis}

We derived the $\mathrm{H} \alpha$ luminosity function (LF) of the $\mathrm{H}$ II regions in each galaxy (for details see Rozas, Beckman \& Knapen 1996) and measured, separately, the diffuse $\mathrm{H} \alpha$ flux for the whole disc, integrating this out to a radius close to $R_{25}$. We corrected this for unresolved $\mathrm{H}$ II regions by extrapolating the statistically incomplete LF below its completeness limit at $\sim 10^{37 \cdot 5} \mathrm{erg} \mathrm{s}^{-1}$ down to $10^{35} \mathrm{erg} \mathrm{s}^{-1}$, using the only complete LF available, that of M31 by Walterbos and Braun (1992). To compute the Lyc flux escaping from the HII regions and available to ionise the diffuse gas we extrapolated the LF, using the slope measured below $L=10^{38 \cdot 6} \mathrm{erg} \mathrm{s}^{-1}$, to higher values, and subtracted off the observed LF. The procedure is illustrated in Figure 1. A brief explanation of the underlying theory is given in Beckman, Rozas \& Knapen (1988, present issue p. 83). A comparison of the available Lyc, and that required to produce the observed diffuse $\mathrm{H} \alpha$ is shown in Table 1. We can see that there is more than sufficient ionising flux available in all four galaxies.

Table 1. Comparison between observed diffuse $\mathrm{H} \alpha$ and available ionising flux predicted from the density bounded H II regions

\begin{tabular}{lcc}
\hline Galaxies & $\begin{array}{c}\text { Diffuse } \\
\text { luminosity }\left(\mathrm{erg} \mathrm{s}^{-1}\right)\end{array}$ & $\begin{array}{c}\text { Available } \\
\text { luminosity }\left(\mathrm{erg} \mathrm{s}^{-1}\right)\end{array}$ \\
\hline NGC157 & $1 \cdot 12 \times 10^{41}$ & $3 \cdot 76 \times 10^{41}$ \\
NGC676 & $6 \cdot 67 \times 10^{40}$ & $1 \cdot 92 \times 10^{41}$ \\
NGC6951 & $6 \cdot 75 \times 10^{40}$ & $2 \cdot 78 \times 10^{41}$ \\
NGC3631 & $7 \cdot 87 \times 10^{40}$ & $1 \cdot 13 \times 10^{41}$ \\
\hline
\end{tabular}

\section{Conclusions}

These results give support to the hypothesis that the diffuse $\mathrm{H} \alpha$ in disc galaxies is produced by Ly continuum escaping from HII regions. This general idea agrees with work by Ferguson et al. (1996) who estimated the diffuse emission at some $40 \%$ of the total $\mathrm{H} \alpha$ produced by the galaxy, and showed a global geometrical association of diffuse emission and emission from the HII regions in the two galaxies they studied. Here we go further and identify the sources as the density bounded regions, showing that they emit enough Lyc to ionise the diffuse medium, and that a significant fraction of the ionising photons produced by a disk galaxy in fact escapes into the intergalactic medium. (Project support: Grant PB94-1107; DGICYT). 

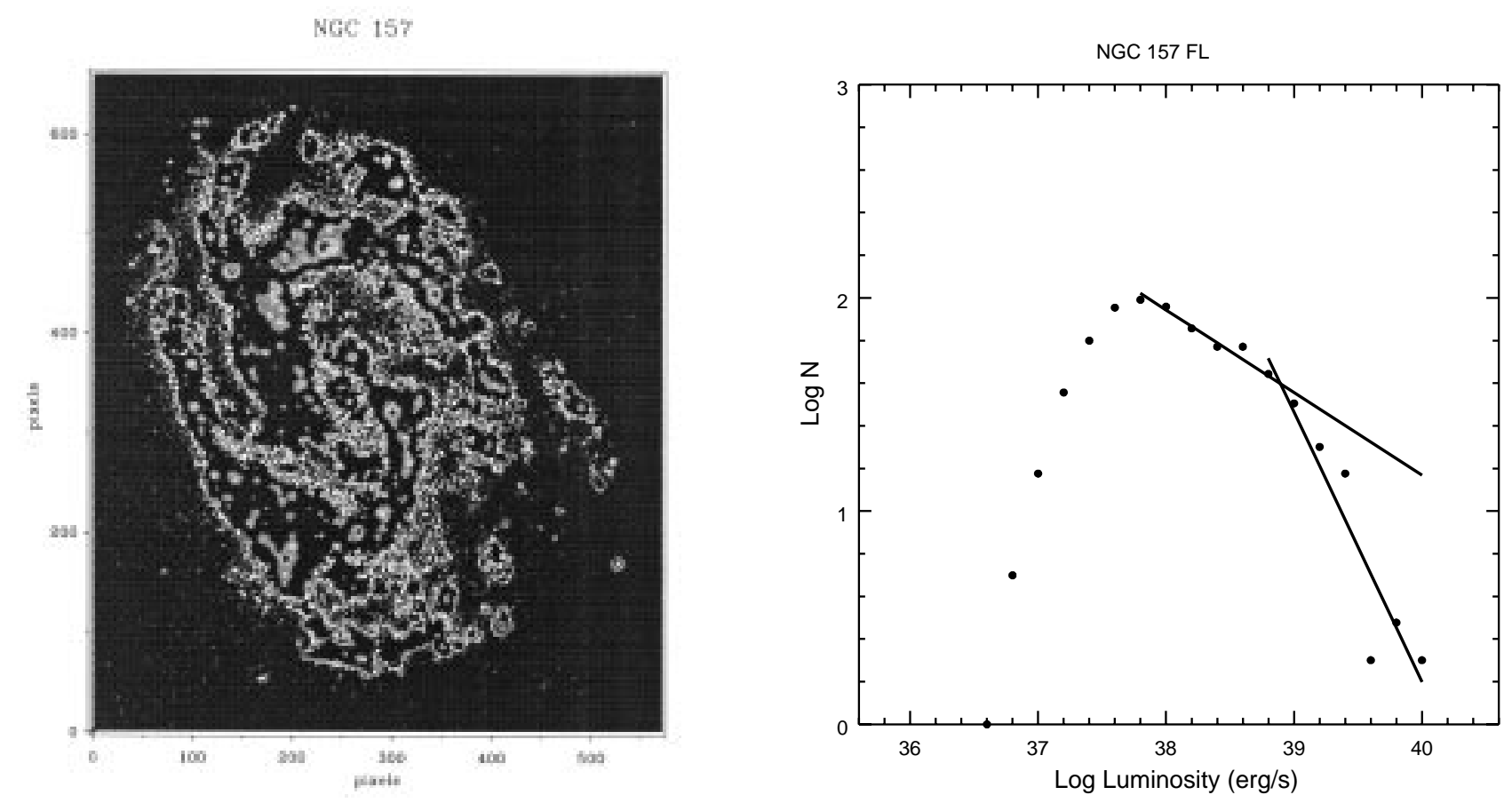

Figure 1-Left: Geometrical match between the observed diffuse $\mathrm{H} \alpha$ and the sites of the luminous regions (density bounded). Right: Procedure for estimating the Lyman continuum flux escaping from the H II regions in four disk galaxies, and available to ionise the diffuse medium. The difference between the extrapolated LF (upper line) and the observed LF from the density bounded high luminosity regions gives the available ionising component.

Beckman, J. E., Rozas, M., \& Knapen., J. H. 1998, PASA, 15,83

Ferguson, A. M., Wyse, R. F., Gallagher, J. S., \& Hunter, D. A. 1996, AJ, 111, 2265
Rozas, M., Beckman, J. E., \& Knapen, J. H. 1996, A\&A, 307,735

Walterbos, R. A. M., \& Braun, R. 1992, A\&AS, 92, 625 